\title{
Severe Chest Wall Toxicity From Cryoablation in the Setting of Prior Stereotactic Ablative Radiotherapy
}

Aadel A. Chaudhuri ${ }^{1}$, Michael S. Binkley ${ }^{1}$, Sonya Aggarwal ${ }^{1}$, Yushen Qian ${ }^{1}$, Justin N. Carter 1 , Rajesh Shah ${ }^{2}$, Billy W. Loo Jr. ${ }^{1}$

1. Department of Radiation Oncology, Stanford University School of Medicine 2. Department of Interventional Radiology, Stanford University School of Medicine

$\square$ Corresponding author: Billy W. Loo Jr., bwloo@stanford.edu

Disclosures can be found in Additional Information at the end of the article

\section{Abstract}

We present the case of a 42 -year-old woman with metastatic synovial sarcoma of parotid origin, treated definitively with chemoradiation, who subsequently developed oligometastatic disease limited to the lungs. She underwent multiple left and right lung wedge resections and left lower lobectomy, followed by right lower lobe stereotactic ablative radiotherapy (SABR), 54 Gy in three fractions to a right lower lobe lesion abutting the chest wall. Two years later, she was treated with cryoablation for a separate right upper lobe nodule abutting the chest wall. Two months later, she presented with acute shortness of breath, pleuritic chest pain, decreased peripheral blood $\mathrm{O} 2$ saturation, and productive cough. A computed tomography (CT) scan demonstrated severe chest wall necrosis in the area of recent cryoablation that, in retrospect, also received a significant radiation dose from her prior SABR. This case demonstrates that clinicians should exercise caution in using cryoablation when treating lung tumors abutting a previously irradiated chest wall.

Note: Drs. Loo and Shah contributed equally as co-senior authors.

Received 12/22/2015

Review began 01/05/2016 Review ended 01/21/2016 Published 02/02/2016

\section{(c) Copyright 2016}

Chaudhuri et al. This is an open access article distributed under the terms of the Creative Commons Attribution License CC-BY 3.0. which permits unrestricted use, distribution, and reproduction in any medium, provided the original author and source are credited.
Categories: Radiation Oncology, Radiology, Oncology

Keywords: stereotactic ablative radiotherapy (sabr), stereotactic body radiation therapy, cryoablation, cryoablation toxicity, radiation related toxicities, chest wall necrosis, synovial sarcoma, metastatic cancer, oligometastasis, interventional oncology

\section{Introduction}

As systemic cancer therapies continue to improve, oncologists are seeing more patients with oligometastatic or oligoprogressive disease, where metastatic disease is well controlled except for in discrete locations. For these cases, we have become more comfortable offering focal therapies, such as surgery, stereotactic ablative radiotherapy (SABR), and/or cryoablation. However, the risk of multimodal local tumor therapy is not fully understood in these scenarios. It is also not always well understood how much time and distance is safe between different treatment modalities. Here, we describe a case of a patient who developed severe chest wall necrosis after cryoablation in the setting of prior SABR to a nearby chest wall-abutting nodule. Informed consent was obtained from the patient for this study.

\section{Case Presentation}

A 40-year-old female with a synovial sarcoma of parotid origin developed lung-only 


\section{Cureus}

oligometastases 17 months after completing her original course of definitive therapy. She underwent chemotherapy, bilateral lung wedge resections, and a left lower lobectomy. Six years after the diagnosis, she presented to our clinic for consideration of stereotactic ablative radiotherapy (SABR) of a right lower lobe oligometastasis abutting the lateral sixth rib. The lesion was round, well-defined, and abutted the right chest wall next to the sixth and seventh ribs (Figure $1 A$ ). We felt that she was a good candidate for lung SABR, and treated her with 54 Gy in three fractions using image-guided radiotherapy and inspiratory breath-hold technique (Figure $1 B$ ). The chest wall at the level of the lateral fifth rib received 10-30 Gy (Figure 1B).

\section{Years before Cryoablation}

A:

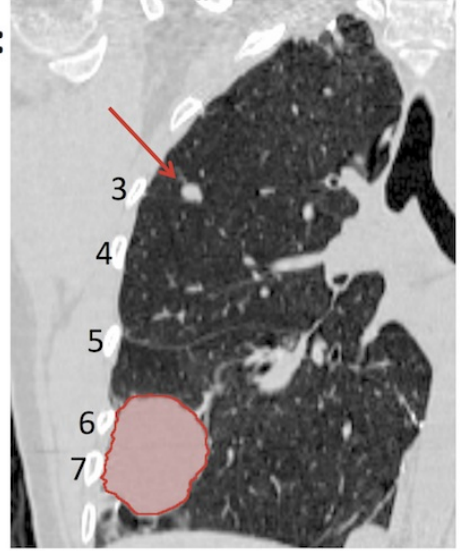

B:

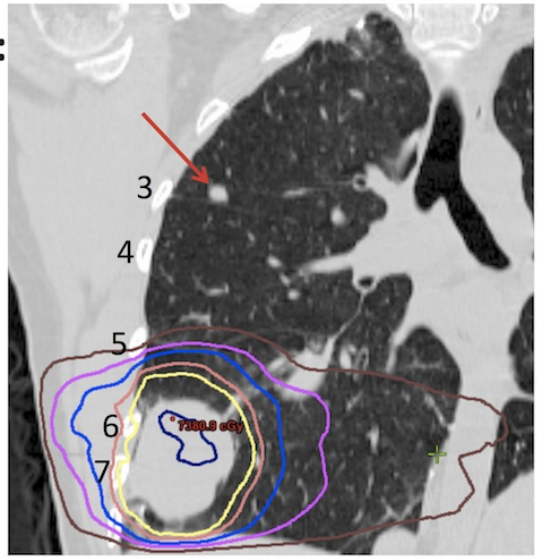

Isodose Levels $70 \mathrm{~Gy}$

$45 \mathrm{~Gy}$

$30 \mathrm{~Gy}$

$20 \mathrm{~Gy}$

$10 \mathrm{~Gy}$

\section{FIGURE 1: Right lower lobe nodule abutting the chest wall treated with SABR (54 Gy in three fractions)}

(A) Right lower lobe nodule highlighted in red. (B) SABR treatment plan with radiotherapy dose depicted as isodose levels. Red arrows indicate right upper lobe nodule subsequently treated two years later with cryoablation. Rib numbers are as indicated. SABR = stereotactic ablative radiotherapy.

Two years after SABR, she developed progression of a separate oligometastatic right upper lung nodule. This had been present two years earlier (Figure $1 A$ ), originally well superior to the lesion previously treated with SABR, but had grown substantially in the interval and was now abutting the right lateral chest wall (Figure $2 A$ ). Because of concerns of cumulative lung parenchymal damage, given her prior course of SABR and multiple surgical resections, she was referred to interventional radiology for consideration of a complementary modality to provide local tumor control. Cryoablation was administered, and the tumor was treated with six cryoprobes with three freeze cycles totaling 20 minutes (Figure $2 B$ ). 


\section{Cureus}

$\underline{\text { Time of Cryoablation }}$

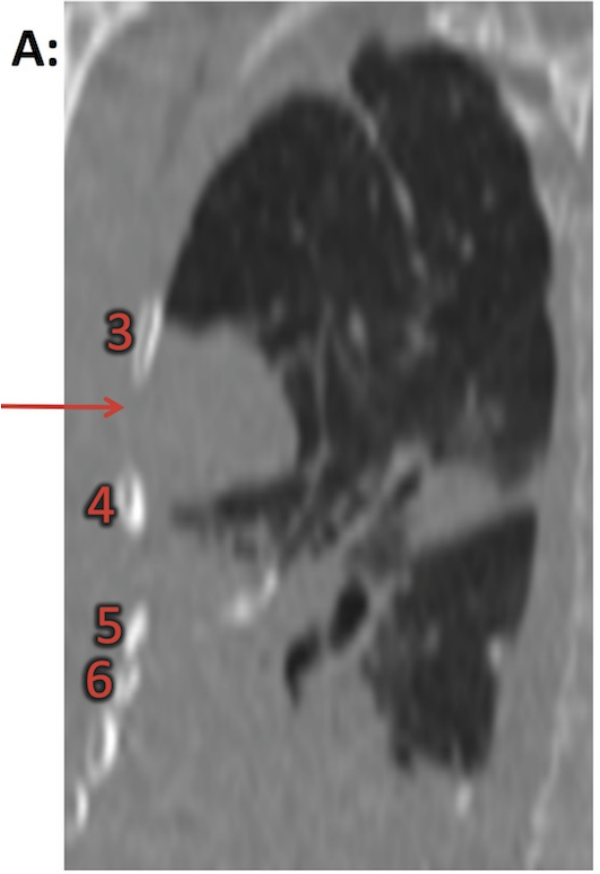

B:

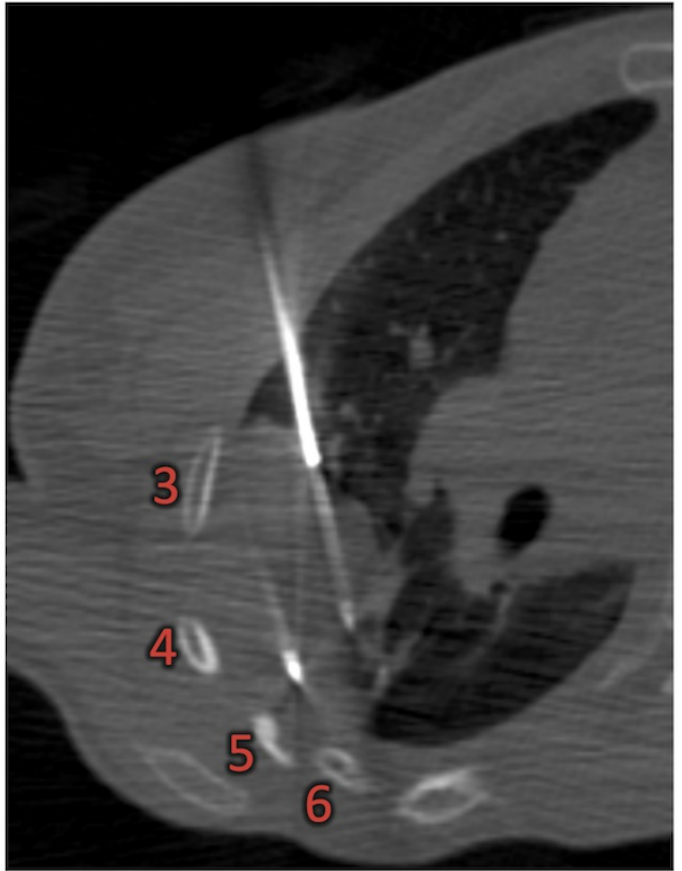

\section{FIGURE 2: Right upper lobe nodule abutting the chest wall} treated with cryoablation

(A) Right upper lobe nodule indicated by red arrow. (B) Axial image with cryoablation probes in place. Rib numbers are as indicated. In retrospect, the right upper lobe nodule that was originally distant from the prior radiation field is now adjacent to a portion of the chest wall that received significant radiation dose because of interval right lower lobe volume loss.

During the procedure, the patient developed hypoxia with $\mathrm{SpO}_{2} 89 \%$, requiring intubation. A CT post-procedure showed extensive hemorrhage involving all lobes of the right lung. The patient recovered after a short ICU stay and was discharged home in stable condition on 1.5-2 L nasal cannula oxygen.

Two months later, the patient was admitted to the hospital with four days of fever, productive cough, shortness of breath, and right-sided pleuritic chest pain. A CT demonstrated a large cavitary lesion, extending from the right peripheral lung through the adjacent right lateral chest wall at the level of ribs three, four, and five into the musculature, with adjacent lobar consolidation (Figure 3). 


\section{Cureus}

2 Months after Cryoablation

A:

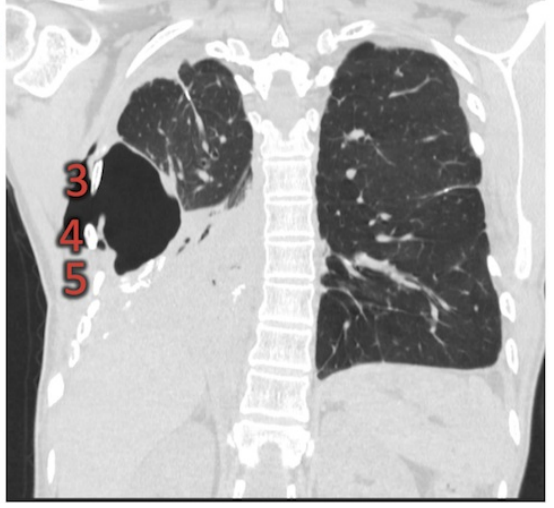

B:

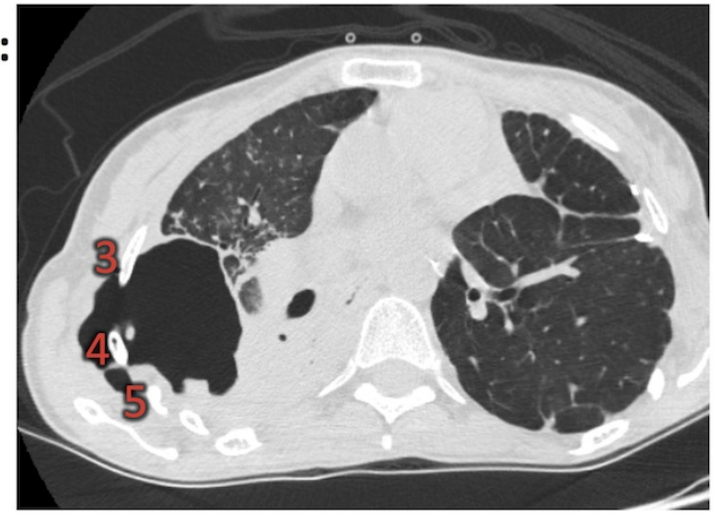

FIGURE 3: Severe right chest wall toxicity two months following cryoablation

A) Coronal and B) Axial views of right-sided cavitary lesion extending through the chest wall past the ribs, with substantial soft tissue destruction and adjacent right lower lobe dense consolidation. Rib numbers are as indicated.

We monitored the patient closely on telemetry, and administered broad-spectrum antibiotics, nasal cannula oxygen, nebulizers, and chest physical therapy. Subsequent CT scans showed the persistence of the large right lung cavitary lesion extending through the right lateral chest wall, but an improvement of right-sided lobar consolidation. Her respiratory symptoms improved, she became afebrile and was discharged from the hospital in stable condition after a five-day inpatient stay, with her previous baseline requirement of 1.5-2 L nasal cannula oxygen. We considered surgical repair of the large chest wall defect, but with substantial concerns about impaired healing due to prior radiotherapy and cryotherapy. She declined surgery in favor of supportive care. Her respiratory symptoms ultimately worsened, and she died six months later.

\section{Discussion}

Studies have shown that SABR of lung tumors near the chest wall can be associated with toxicity, such as chest wall pain and rib fractures [1-2]. However, severe toxicity, such as chest wall necrosis, is extremely rare, limited to a case report by Woody, et al. of a case of chest wall abscess and bronchopleural fistula 17 months following SABR (50 Gy in five fractions) of a pleural-based left lower lobe non-small cell lung cancer [3]. Factors contributing to chest wall toxicity following SABR include tumor size, tumor location, treatment volume, chest wall radiation dose, and potentially bone density and body habitus [2, 4-6].

Similarly, severe chest wall toxicity after cryoablation, even of tumors abutting the chest wall, is extremely rare [7]. Cryoablation has been shown in isolated cases to cause irreversible thermal damage [8]. Ito, et al. showed in their retrospective cohort that $35 \%$ of lung cryoablation zones cavitate after treatment, but that $96 \%$ of these resolve by six months [9].

In our patient's case, since we treated a right lower lobe oligometastasis directly abutting the chest wall with SABR $54 \mathrm{~Gy}$, we elected to use a complementary modality to treat a separate right upper lobe nodule two years later. The new nodule was treated with cryoablation, which was followed by severe chest wall toxicity, including massive soft tissue necrosis. In retrospect, although the right upper lobe lesion treated with cryotherapy was originally far outside the treatment field of the right lower lobe lesion treated with SABR, over time, post-SABR fibrosis 
and volume loss led to the right upper lobe lesion moving inferiorly and abutting the region of the chest wall within the prior radiation field at the level of ribs five and six (Figure 2). We hypothesize that the combination of cryotherapy involving a previously irradiated area of chest wall led to the development of unexpected severe chest wall toxicity. This phenomenon has not been previously reported per our review of the literature.

There is no standard set of dosimetric or cryotherapy constraints when using these complementary modalities. Thus, it is difficult to predict whether a patient might experience a severe toxicity as our patient unfortunately did. However, it is well-known that radiotherapy impairs wound healing. This case illustrates that we should exercise caution when performing cryoablation in the setting of prior SABR for lesions abutting the chest wall. We cannot exclude the possibility that cryoablation overlapping with less intensive palliative radiotherapy doses could also be toxic.

\section{Conclusions}

We present here a report of severe chest wall necrosis following cryoablation of a nodule abutting a portion of chest wall that received a significant dose of radiation from a prior course of stereotactic ablative radiotherapy to a separate lesion. We recommend caution when combining these two modalities for treatment of pulmonary tumors abutting the chest wall.

\section{Additional Information \\ Disclosures}

Human subjects: Consent was obtained by all participants in this study. Conflicts of interest: In compliance with the ICMJE uniform disclosure form, all authors declare the following:

Payment/services info: All authors have declared that no financial support was received from any organization for the submitted work. Financial relationships: Billy W. Loo Jr. declare(s) a grant and personal fees from Varian Medical Systems. Billy W. Loo Jr. has received research grant funding and payment for educational lectures from Varian Medical Systems. Billy W. Loo Jr. declare(s) a grant from Raysearch Laboratories. Billy W. Loo Jr. has received research grant funding from Raysearch Laboratories. Other relationships: All authors have declared that there are no other relationships or activities that could appear to have influenced the submitted work.

\section{References}

1. Stephans KL, Djemil T, Tendulkar RD, Robinson CG, Reddy CA, Videtic GM: Prediction of chest wall toxicity from lung stereotactic body radiotherapy (SBRT). Int J Radiat Oncol Biol Phys. 2012, 82:974-980. 10.1016/j.ijrobp.2010.12.002

2. Dunlap NE, Cai J, Biedermann GB, Yang W, Benedict SH, Sheng K, Schefter TE, Kavanagh BD, Larner JM: Chest wall volume receiving > 30 Gy predicts risk of severe pain and/or rib fracture after lung stereotactic body radiotherapy. Int J Radiat Oncol Biol Phys. 2010, 76:796-801. 10.1016/j.ijrobp.2009.02.027

3. Woody NM, Djemil T, Adelstein DJ, Mason DP, Rice TW, Videtic GM: Severe local toxicity after lung stereotactic body radiation therapy: lesional abscess leading to bronchocutaneous fistula requiring surgical marsupialization. J Thorac Oncol. 2010, 5:1874-1875.

10.1097/JTO.0b013e3181f13699

4. Bongers EM, Haasbeek CJ, Lagerwaard FJ, Slotman BJ, Senan S: Incidence and risk factors for chest wall toxicity after risk-adapted stereotactic radiotherapy for early-stage lung cancer. J Thorac Oncol. 2011, 6:2052-2057. 10.1097/JTO.0b013e3182307e74

5. Thibault I, Chiang A, Erler D, Yeung L, Poon I, Kim A, Keller B, Lochray F, Jain S, Soliman H, Cheung P: Predictors of chest wall toxicity after lung stereotactic ablative radiotherapy . Clin Oncol. 2016, 28:28-35. 10.1016/j.clon.2015.06.009

6. Welsh J, Thomas J, Shah D, Allen PK, Wei X, Mitchell K, Gao S, Balter P, Komaki R, Chang JY: 


\section{Cureus}

Obesity increases the risk of chest wall pain from thoracic stereotactic body radiation therapy . Int J Radiat Oncol Biol Phys. 2011, 81:91-96. 10.1016/j.ijrobp.2010.04.022

7. de Baere T, Tselikas L, Woodrum D, Abtin F, Littrup P, Deschamps F, Suh R, Aoun HD, Callstrom M: Evaluating cryoablation of metastatic lung tumors in patients-safety and efficacy: the ECLIPSE trial-interim analysis at 1 year. J Thorac Oncol. 2015, 10:1468-1474. 10.1097/JTO.0000000000000632

8. Rhim H, Dodd GD III, Chintapalli KN, Wood BJ, Dupuy DE, Hvizda JL, Sewell PE, Goldberg SN: Radiofrequency thermal ablation of abdominal tumors: lessons learned from complications . Radiographics. 2004, 24:41-52. 10.1148/rg.241025144

9. Ito N, Nakatsuka S, Inoue M, Yashiro H, Oguro S, Izumi Y, Kawamura M, Nomori H, Kuribayashi S: Computed tomographic appearance of lung tumors treated with percutaneous cryoablation. J Vasc Interv Radiol. 2012, 23:1043-1052. 10.1016/j.jvir.2012.04.033 